\title{
Marcellin Jobard et le Musée royal de l'Industrie de Bruxelles
}

Marcellin Jobard and the Musée royal de l'Industrie in Brussels

\section{Marie-Christine Claes}

\section{(2) OpenEdition}

\section{Journals}

Édition électronique

URL : http://journals.openedition.org/artefact/630

DOI : $10.4000 /$ artefact. 630

ISSN : 2606-9245

Éditeur :

Association Artefact. Techniques histoire et sciences humaines, Presses universitaires du Midi

Édition imprimée

Date de publication : 15 juin 2017

Pagination : $59-75$

ISBN : 978-2-7535-6525-8

ISSN : 2273-0753

Référence électronique

Marie-Christine Claes, « Marcellin Jobard et le Musée royal de l'Industrie de Bruxelles », Artefact [En ligne], 5 | 2016, mis en ligne le 15 novembre 2017, consulté le 04 mars 2020. URL : http:// journals.openedition.org/artefact/630 ; DOI : https://doi.org/10.4000/artefact.630 


\section{Marcellin Jobard et le Musée royal de I'Industrie de Bruxelles}

Marie-Christine CLAES*

\section{Résumé}

En 1826, est créé à Bruxelles un « Conservatoire des Arts et Métiers ». Le premier directeur, Jacob Canzius-Onderdewijngaart, constructeur d'instruments scientifiques à Delft, y intègre sa propre collection. À partir de 1841, Marcellin Jobard, directeur de journaux, ancien lithographe et premier photographe belge, dirige le désormais "Musée royal de l'industrie » et publie son Bulletin, qui touche un large public belge et étranger. Hélas! après sa mort en 1861, on vend une partie des collections et le reste est annexé à une école industrielle. La plupart des objets ont aujourd'hui disparu. Heureusement, l'astronome Adolphe Quetelet, membre de la commission administrative du musée, avait fait déposer en 1841 à l'Observatoire royal de Belgique à Uccle (Bruxelles) une dizaine des plus beaux instruments, aujourd'hui présentés par les Musées royaux d'art et d'histoire de Bruxelles. Reste aussi un catalogue de trois mille pièces, dressé par Édouard Mailly en 1846, qui permettra peut-être de reconstituer virtuellement l'un des plus beaux musées techniques d'Europe au xixe siècle.

Mots-clés : Bruxelles, Canzius-Onderdewijngaart (Jacob), catalogue virtuel, instruments, Jobard (Marcellin), musée.

\section{Abstract. Marcellin Jobard and the Musée royal de I'Industrie in Brussels}

When the "Conservatoire des Arts et Métiers » was founded in Brussels in 1826, its founding director, Jacob Canzius-Onderdewijngaart, a manufacturer of scientific instruments in Delft,

\footnotetext{
*. Docteur en histoire de l'art, Marie-Christine Claes est scientifique au département Documentation de l'Institut royal du patrimoine artistique (établissement scientifique fédéral belge) et responsable des thésaurus de l'Infothèque. Co-auteur avec Steven F. Joseph et Tristan Schwilden du Directory of photographers in Belgium, 1839-1905 [http://www.directorybelgianphotographers.be/], elle a publié plusieurs articles sur l'histoire de la photographie en Belgique. Bibliographie en ligne : [http://balat.kikirpa.be/pi/10161]. Contact : [marie-christine.claes@kikirpa.be].
} 
brought his own collection into the institution. In 1841, Marcellin Jobard, a newspaper editor, former lithographer, and Belgium's first photographer, became the director of Musée royal de l'Industrie, a successor institution, and published a Bulletin, which had a wide readership in Belgium and abroad. After Jobard's death in 1861, part of the collections was sold and the rest given to an industrial school in Brussels. Although, most objects remain lost to history, a dozen of the finest instruments were deposited in 1841 at the royal astronomical observatory of Belgium located in Uccle (Brussels) by the astronomer Adolphe Quetelet, member of the administrative commission of the museum and these are now held at the Royal Museums of Art and History in Brussels. There is also a catalogue of 3000 objects, compiled by Édouard Mailly in 1846, which is a precious source for current attempts to reconstruct virtually one of Europe's finest technical museums of the 19th century.

Keywords : Brussels, Canzius-Onderdewijngaart (Jacob), instruments, Jobard (Marcellin), museum, virtual catalogue.

Pendant les vingt dernières années de sa vie, de 1841 à 1861, Marcellin Jobard a été directeur du Musée royal del'Industrie de Bruxelles (figure 1). Fondateur en 1820 du premier établissement lithographique belge important et premier photographe belge dès septembre 1839, Jobard a également joué un rôle marquant dans le développement industriel de la Belgique sous le règne (1831-1865) du premier roi des Belges, Léopold I ${ }^{\text {er }}$. Jobard est un modèle du savant du XIX ${ }^{\mathrm{e}}$ siècle, époque où l'industrialisation stimule l'esprit technique et où la somme de connaissances permet encore aux chercheurs d'être " généralistes » et de s'intéresser à des domaines très variés ${ }^{1}$.

Né en 1792 à Baissey (Haute-Marne), Marcellin Jobard devient géomètre en Hollande sous l'Empire dans la région de Groningue. Après les Cent-Jours, il est rappelé par le gouvernement hollandais pour poursuivre ses travaux en raison de ses aptitudes en mathématiques et est envoyé pour arpenter dans la région de Maastricht. En 1816, il obtient la grande naturalisation². Mais, en 1819, il décide de démissionner et de s'installer à Bruxelles pour se lancer dans une carrière lithographique. Il commence en s'associant avec l'imprimeur LouisJules-Michel Weissenbruch (1772-1851) pour l'illustration des Annales générales des sciences physiques. La première mention de ce projet paraît dans un quotidien bruxellois, L'Oracle, le 13 avril 1819 :

« Notre Belgique ne se sera pas inutilement montrée hospitalière : M. Drapiez ${ }^{3}$, chimiste et naturaliste français, auteur de tableaux minéralogiques justement estimés, jeté, par suite des derniers orages politiques, dans notre patrie, s'y est établi et y a transporté ses riches et précieuses collections; le colonel Bory de Saint-Vincent ${ }^{4}$, ci-devant de la première classe de l'Institut, compris dans l'ordonnance du 24 juillet, y fait, dit-on, venir ses herbiers et les minéraux qu'il a recueillis dans ses lointains voyages. Ces savans [sic] se sont, à ce qu'on assure, associés à notre illustre compatriote, 


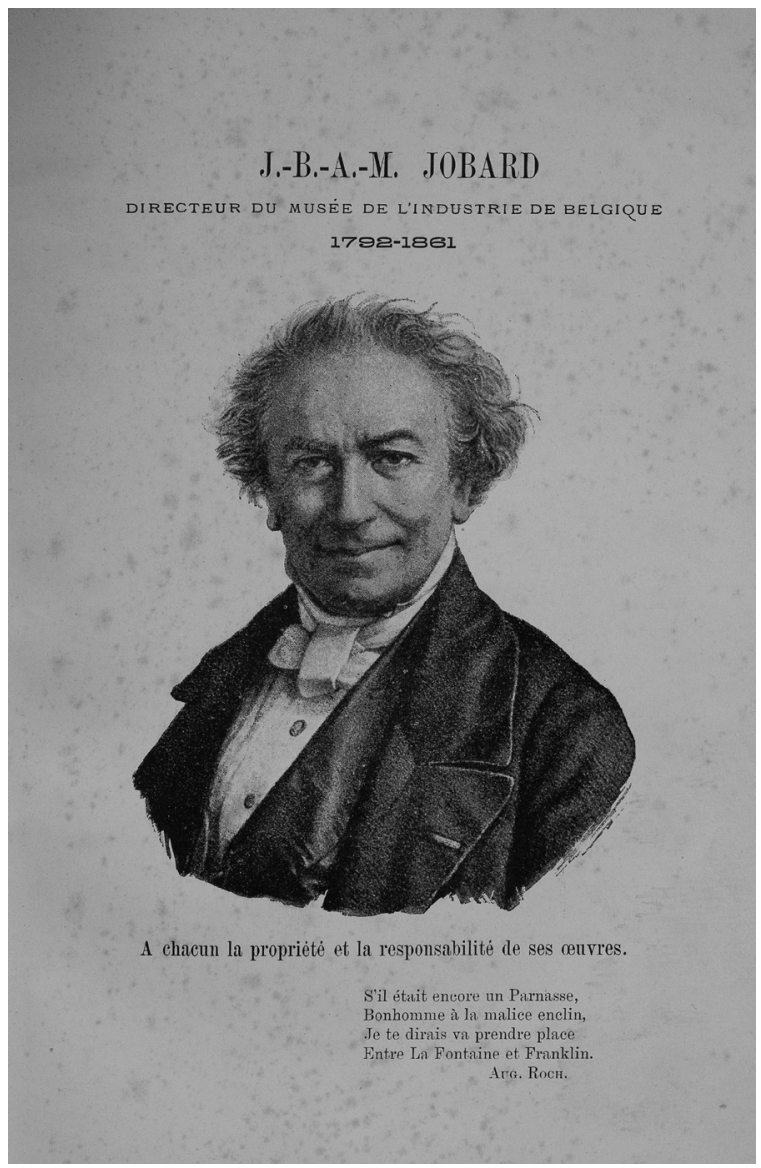

Fig. 1. - Portrait de Marcellin Jobard en frontispice de l'ouvrage de son petit-neveu, Henri Raclot, Brevets d'invention, Bruxelles, Imprimerie des Travaux publics, 1897

(Coll. Steven F. Joseph. @ IRPA, Bruxelles, cliché X022012).

M. Van Mons ${ }^{5}$, professeur de chimie à l'ancienne et célèbre université de Louvain, et vont publier, avec M. Weissenbruch, un ouvrage intitulé : Annales des sciences naturelles. Cet ouvrage paraîtra de mois en mois, par cahiers de sept feuilles, ornés de quatre belles planches enluminées. On assure que des savans [sic] du premier ordre et de tous les pays s'intéressent à cette entreprise, dont le Prospectus paraîtra, dit-on, vers la fin du mois (Article communiqué).»
D'emblée Jobard allie sciences, techniques et images : la première planche de ce périodique est un portrait d'Alexandre von Humboldt. Le lithographe rencontre le naturaliste, avec qui il restera en contact toute sa vie. Les Annales générales des sciences physiques vont mettre Jobard en contact avec de nombreux savants d'Europe et même des États-Unis. C'est le début d'un véritable réseau de relations qui sera pour lui d'une importance capitale. 


\section{Le cabinet de physique du Musée de Bruxelles}

L'imprimerie Weissenbruch est installée rue du Musée. Le Musée de Bruxelles est en fait un ensemble de musées hébergé dans " l'ancienne Cour »; c'est ainsi que l'on appelle alors le palais de Charles de Lorraine (17121780), beau-frère de l'impératrice MarieThérèse, qui fut gouverneur des Pays-Bas sous la période autrichienne. Protecteur des sciences et expérimentateur, Charles de Lorraine avait installé des cabinets de physique et de sciences naturelles dans son palais de Bruxelles. Il avait également implanté des manufactures aux environs de la ville, notamment une fabrique de papiers peints à Tervuren. À sa mort, ses collections sont dispersées : certains objets sont pris par son neveu, l'empereur Joseph II, quelques instruments sont donnés à l'Observatoire de Vienne, d'autres sont vendus et quelques objets restent à Bruxelles, car ils sont offerts à l'Académie impériale et royale des sciences et belles lettres de Bruxelles $^{6}$. La ville devient propriétaire du palais dont elle fait un musée où des cabinets de physique et de sciences naturelles sont reconstitués.

Pendant la période française, le conservateur des collections est Adrien Dekin (1768-1823), ancien élève de Daubenton au Muséum d'histoire naturelle de Paris. Professeur à l'École centrale de Bruxelles sous la période française, il reste en fonction après le traité de Vienne qui rattache la Belgique à la Hollande ${ }^{7}$. Nul doute que Jobard ait visité à plusieurs reprises le "cabinet Dekin ", car les deux hommes se connaissaient. Dekin, « de l'Institut royal des Pays-Bas ", collabore aux Annales avec un article "sur une dent molaire d'éléphant fossile, découverte dans les environs de Bruxelles", que Jobard lithographie d'après un dessin de Bory de Saint-Vincent ${ }^{8}$. Un an avant la mort de Dekin, un « catalogue des instruments de physique, d'optique et de mathématiques déposés au cabinet de Bruxelles » est établi. L'inventaire est terminé par une mention manuscrite de Dekin : "Je certifie que tous les instruments existant au Cabinet de physique du Musée au 2 août 1822, se trouvent repris au présent catalogue. Bruxelles le 2 août 1822 A. Dekin. »

Ce précieux document ${ }^{9}$ comporte 372 numéros, mais, certains concernant plusieurs objets, il en recense en tout 492, notamment des instruments de mesure ou d'optique, des instruments destinés aux expériences électriques, mais aussi à la fantasmagorie. Les archives contiennent également de la correspondance de Dekin, dont une facture du fabricant parisien Pixii :
« Fourni à Monsieur Delkin [sic]
Conservateur du Cabinet de
Physique de la Ville de Bruxelles
21 décembre 1818
Un aéromètre à pompe pour
l'équilibre des liqueurs
Un aéromètre universel
10.00
Le mécascope [sic] solaire de
Monsieur Charles, complet 170.00
Ludion dans le vide
Florins des Pays-Bas
212.00
Pour acquit Pixii »

Le «mécascope » se retrouve dans l'inventaire de 1822 sous la rubrique lumière et porte le numéro 316 : "Mégascope 
solaire de $\mathrm{M}^{\mathrm{r}}$ Charles, composé d'une platine en cuivre portant 3 tuyaux mobiles à frottements, les uns dans les autres, et garnis de verres objectifs, plus
2 miroirs plans, montés sur des genoux en cuivre, servant à éclairer l'objet, et un porte-objet mobile, pour placer audehors de la chambre. »

\section{Du Conservatoire des arts et métiers de Bruxelles au Musée royal de l'Industrie}

En décembre 1826, le roi des Pays-Bas Guillaume Ir crée à Bruxelles un Musée des sciences et des lettres, qui fermera en 1834 à la création de l'Université libre. Les cabinets de sciences naturelles et de physique en constituent la partie scientifique, tandis que les lettres sont mises à l'honneur par des cours. Des leçons de sciences sont également données, notamment des cours de chimie par CharlesÉtienne Guillery (1791-1861 $)^{10}$. Le souverain crée aussi un "Conservatoire des Arts et Métiers »- la dénomination montre que l'institution est calquée sur le modèle parisien. Pendant une dizaine d'années, les dénominations de ces musées seront très fluctuantes, " arts et sciences ", " sciences et lettres », " arts et métiers ", « industrie ». Une certaine confusion semble régner, même dans les documents administratifs.

Le roi place à la direction $\mathrm{du}$ Conservatoire un constructeur d'instruments scientifiques de Delft, Canzius-Onderdewijngaart (1771-1838). Celui-ci apporte à Bruxelles sa collection qui comprend des instruments de Musschenbroeck, Leeuwenhoek et van Marum, pour les intégrer au musée, et achète le cabinet van Renswoude. Malheureusement, en janvier 1827, un mois après les arrêtés de création des musées, un incendie ravage une partie du palais de Charles de Lorraine et les collections du Musée de l'Industrie doivent être abritées dans la maison Mosselman, Courte rue des Longs Chariots ${ }^{11}$, qui a été louée dans le but d'y remiser le musée en attendant mieux ${ }^{12}$.

Cette année-là, Jobard a lancé un journal satirique, Le Manneken. Il intervient à propos du musée (des sciences et des lettres) dès 1827, pour regretter l'absence d'une publication et propose d'y suppléer :

«Musée des sciences et des arts

Le bienfait de cette nouvelle institution est si grand qu'on doit regretter de le voir borné à la seule ville de Bruxelles; nous pensons avec les professeurs les plus distingués de ce Musée, qu'un journal fidèle à présenter le résumé des principaux cours publics, ferait participer à leurs avantages le royaume des Pays-Bas, y répandrait le goût de l'instruction, et seconderait ainsi les intentions d'un monarque ami des lumières.

Un de nos rédacteurs sera donc chargé de rendre compte des séances du Musée, et de servir d'écho aux doctrines des professeurs; ses observations, quelle qu'en soit la 
nature, étant dictées par l'amour des lettres, ne s'écarteront pas des règles de convenance et d'urbanité13. ${ }^{13}$

Deux mois plus tard, Jobard consacre un article au Conservatoire et dévoile déjà sa vision de l'institution : l'information des industriels et la formation de techniciens.

«Industrie

Conservatoire des arts et métiers ${ }^{14}$.

Un conservatoire des arts et métiers s'organise en ce moment à Bruxelles, sous la protection de S. M. et par les soins éclairés de M. Canzius. [...]

Nous croyons qu'un conservatoire ne doit être que le complément d'une école industrielle; c'est là que viendront s'essayer au toucher les élèves que l'étude des théories a préparés; ce sera pour ainsi dire, le mordant qui doit fixer la teinture de leurs connaissances, qui sans cela se dissipent rapidement.

Il faudrait en outre que l'établissement fût organisé de manière à pouvoir satisfaire à toutes les demandes des manufacturiers du royaume, soit pour leur procurer les plans de machines dont ils auraient besoin, soit pour leur faire exécuter des modèles, et leur fournir même toutes les machines existantes dans le pays ou à l'étranger.

Pour cela l'administration entretiendrait un agent dans les principaux foyers de l'industrie, surtout à Londres.

L'administration aurait soin de se tenir au courant de tout ce qui existe de neuf et de tout ce qui se publie en mécanique; l'administration se formerait une bibliothèque de tous les ouvrages anciens de technologie, et s'abonnerait aux recueils de ce genre qui s'impriment journellement; des employés seraient chargés de donner des renseignements sur tout ce qu'on pourrait leur demander touchant les inventions existantes; là l'homme inérudit [sic], que son génie pousse vers la mécanique, viendrait exposer ses idées, pour les voir combattre si elles sont fausses, ou encouragées si elles offrent quelque degré d'utilité.

Tous les plans et modèles exécutés par des élèves de l'établissement seraient payés par les demandeurs, et contribueraient à couvrir les frais du conservatoire.

Un conseil des professeurs et principaux élèves serait formé, pour entendre l'exposé de toutes les nouvelles inventions; après un mûr examen l'invention serait rejetée, modifiée ou adoptée; des plans, des modèles seraient dans ce cas exécutés aux frais de l'établissement, et les non-succès seraient amplement couverts par la mise en lumière des inventions utiles, qui seraient probablement mortes avec leurs inventeurs, car il est rare que les occupations, les moyens pécuniaires, les études et la persévérance d'homme, ainsi que les circonstances concourent à pousser à bonne fin des créations souvent précieuses pour l'humanité.

Nous reviendrons sur ce sujet qui est digne d'attirer l'attention de tous les amis de la perfectibilité de notre espèce $^{15}$. » 
Dans son périodique, Jobard ne manque jamais de signaler quand le modèle d'une machine décrite dans un de ses articles existe " au musée de l'état pour les arts et les industries nationales ", comme, par exemple, l'appareil à creuser les canaux décrit aux p. 346-347 du tome 3,1830 , et illustré par une lithographie dessinée par Jobard lui-même. Il publiera également des informations sur ses propres inventions, parfois sous un pseudonyme (figure 2).

En 1829, Le Manneken perd son ton caustique et devient L'Industriel ou Revue des revues, et Jobard y publie un texte sur le musée, le comparant à celui de Paris :
«Plusieurs personnes entendront pour la première fois la nouvelle de l'existence d'un conservatoire à Bruxelles; mais leur surprise augmentera de beaucoup si elles parviennent à le découvrir et à le visiter : ce ne sont point ces machines colossales, rongées de rouille, ces immenses et vieux métiers à filer détraqués, remplis d'araignées et de poussière, entassés dans les vastes halles de l'abbaye St-Denis [sic pour le prieuré de Saint-Martindes-Champs] ; mais ici on trouve au contraire, rassemblées dans de jolies salles entretenues avec la propreté

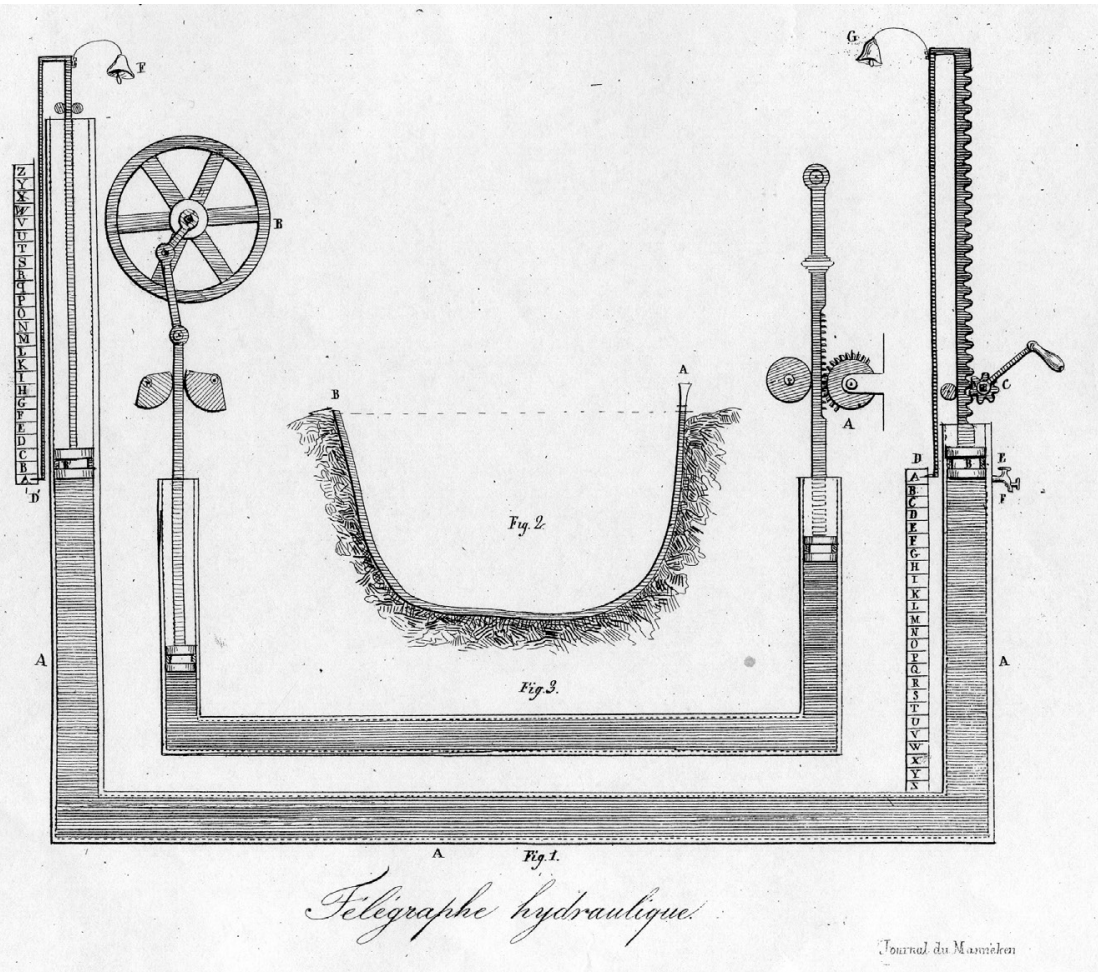

Fig. 2. - « Thélégraphe [sic] hydraulique pouvant jouer la nuit et le jour, et communiquant la pensée instantanément à toutes distances, sans positions intermédiaires et avec une grande économie dans les constructions; de l'invention de M. Nilecram Darobi citoyen du Kentuki », planche lithographiée, Le Manneken, $\mathrm{n}^{\circ}$ 18, 27 septembre 1827 [Nilecram Darobi est presque l'anagramme de Marcellin Jobard] (Coll. Université catholique de Louvain, Louvain-la-Neuve. Cliché de l'auteur). 
recherchée des Hollandais, sur un modèle petit, mais assez grand pour ne rien laisser à désirer dans les plus petits détails, toutes les machines colossales qui demanderaient trop d'emplacement : une petite salle, par exemple, contient les modèles de toutes les espèces de machines à vapeur connues, leur mécanisme est si soigné qu'elles peuvent fonctionner parfaitement sous vos yeux avec la flamme d'une bougie; l'œil le moins exercé en embrasse et en comprend le mécanisme beaucoup mieux qu'il ne le ferait sur une grande machine. Quant aux instrumens [sic] de petite dimension, ils y figurent à leur échelle et dans un degré de perfection rare : rien de plus complet que les salles de physique, de catoptrique et de dioptrique; c'est là qu'existe la plus grande et la plus puissante machine électrique de l'Europe, construite $\mathrm{d}$ 'après le système du célèbre professeur Van Marum ${ }^{16}$. »

Place du Musée, deux nouvelles ailes sont construites. La première, qui doit devenir le Palais de l'Industrie, est inaugurée en 1830 (figures 3 et 4). Mais les activités sont interrompues en 1830 par la révolution belge. Canzius retourne en Hollande, où il finit tristement ses jours suite à un litige l'opposant à l'État belge au sujet de ses collections ${ }^{17}$.

Jobard est lui aussi victime de la révolution: son établissement lithographique fait faillite et il se reconvertit dans l'industrie. Il part à Verviers, ville dont

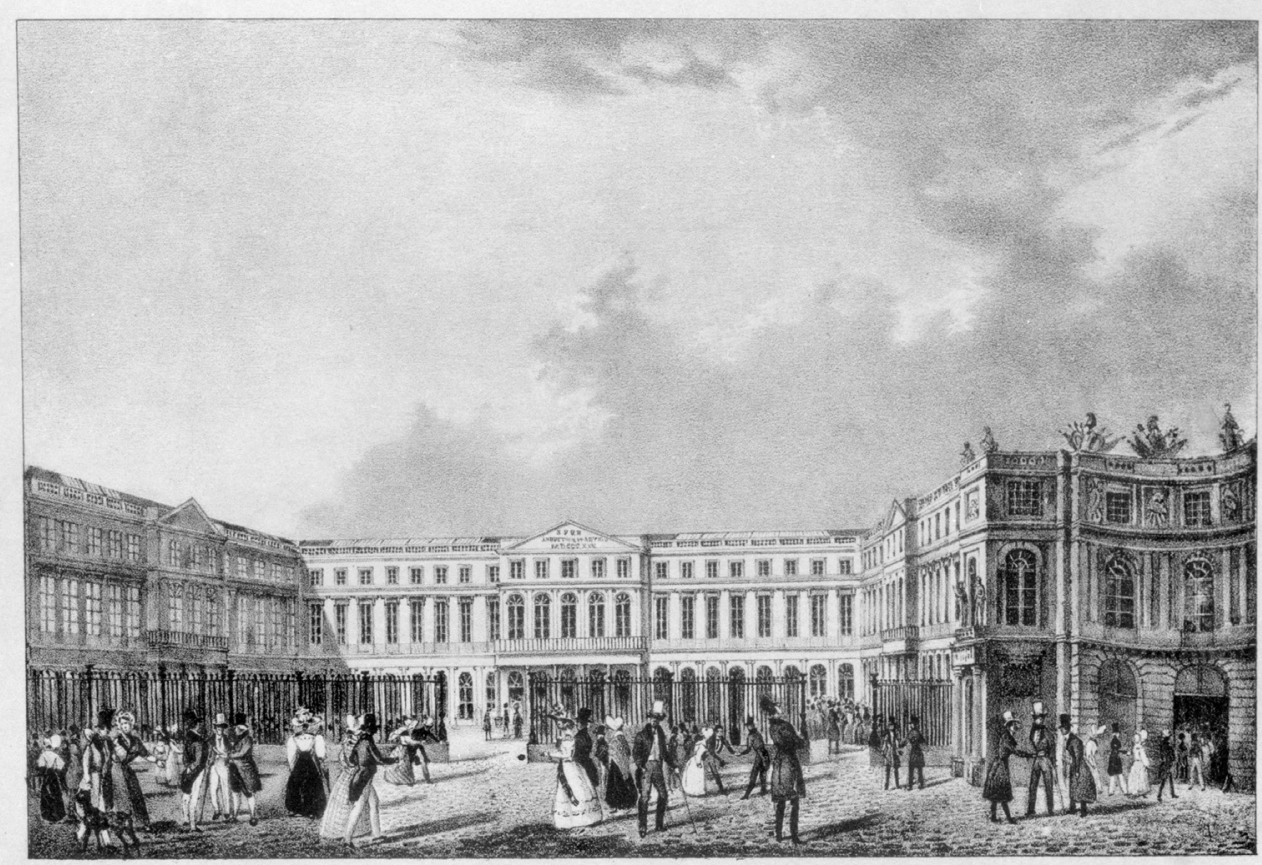

Fig. 3. - Ed. Loose, « Palais de l'Exposition des produits de l'Industrie nationale », lithographie, s.d. [1830], éditée par Fietta Frères, rue de la Colline, 295, à Bruxelles ( IRPA, Bruxelles, cliché M279738). 
est originaire Gérard-Joseph Christian (1776-1832), directeur du Conservatoire des arts et métiers de Paris de 1817 à 1830. Le Verviétois est une gloire locale ${ }^{18}$ et est peut-être déjà alors devenu un modèle pour Jobard. Celui-ci passe une année dans la cité lainière où il construit des machines à vapeur.

Quand Jobard revient à Bruxelles, le musée est toujours dans la maison Mosselman. Un directeur éphémère est en poste de 1831 à $1832^{19}$ : Napoléon Bauwens, le fils du célèbre Lieven Bauwens qui avait introduit les filatures mécaniques sur le continent. Napoléon Bauwens (1800-1859) a obtenu sa nomination grâce à des recommandations émanant de sommités fran- çaises, Clément Desormes, "professeur de chimie au Conservatoire des Arts et Métiers ", "C. P. C. Molard, fondateur du Conservatoire des Arts et Métiers ", le baron Edme Jomard, "Membre de l'Institut », cartographe, et le zoologiste Geoffroy Saint-Hilaire ${ }^{20}$.

En 1832, le Palais de l'Industrie n'est toujours pas disponible : à la suite d'une épidémie de choléra, il devient un hôpital. On retrouve Jobard dans le combat contre l'épidémie : il est envoyé à Lille comme commissaire sanitaire. Puis il entre en contact avec les saint-simoniens, dont il devient le propagandiste en Belgique; mais les querelles internes et les démêlés judiciaires du mouvement qui aboutiront à la mise sous scellés de la maison

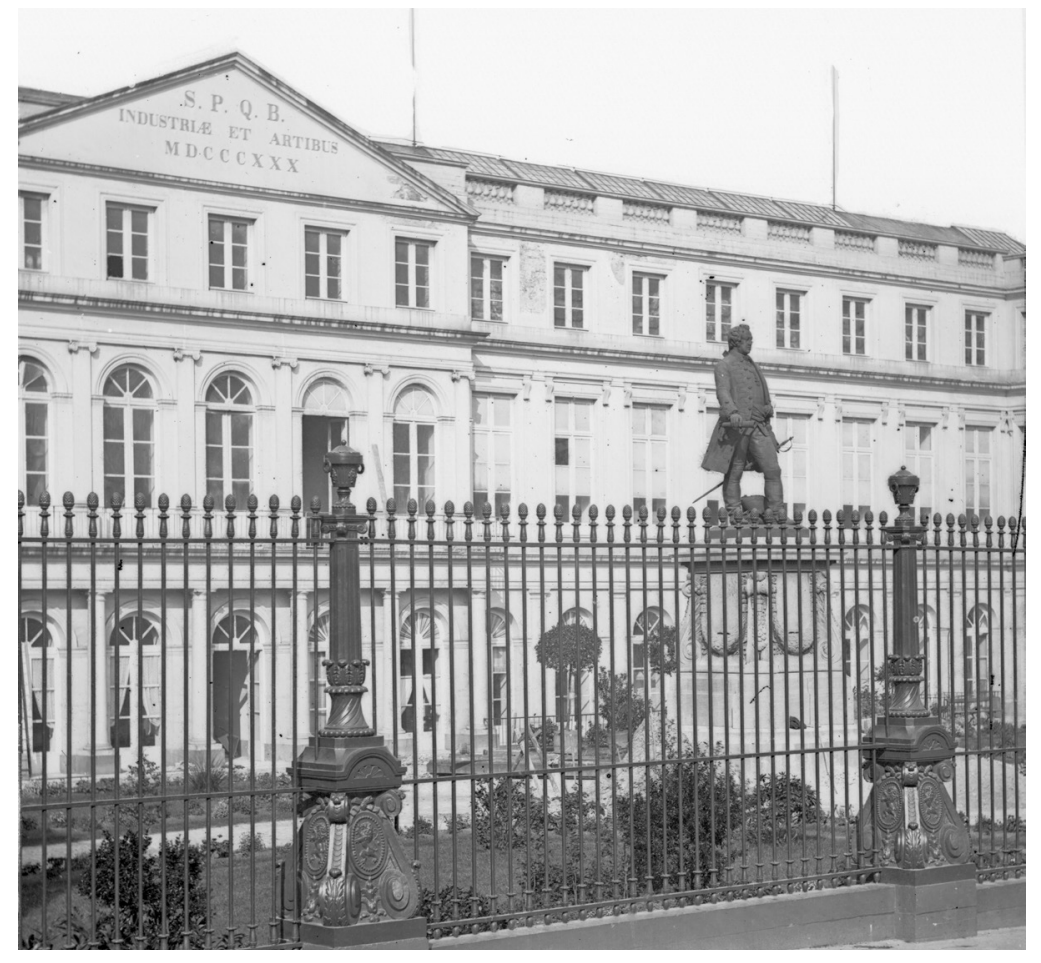

Fig. 4. - Anonyme, Façade du Palais de l'Industrie, s.d. [avant 1880, date du placement d'un nouveau fronton sculpté] (@ IRPA, Bruxelles, cliché A124849, scan d'une moitié de négatif stéréoscopique 13×18 au collodion). La statue de Charles de Lorraine, inaugurée le 25 novembre 1848, a dû être déplacée pour la construction du musée d'art moderne, construit sous la cour. 
des saint-simoniens à Ménilmontant sont détaillés dans la presse belge et le saintsimonisme est tourné en ridicule ${ }^{21}$. On est loin du beau rêve initial de l'association des travailleurs et de l'amélioration des classes défavorisées qui seul intéressait Jobard. « La doctrine n'est qu'un livre de plus, une utopie de plus " (c'est Jobard qui souligne ${ }^{22}$ ). Bien que de courte durée, un an à peine, le militantisme saintsimonien de Jobard a cependant pour lui une importance considérable et le marque à long terme : il en retiendra toujours les idées visionnaires et la capacité d'anticipation.

En 1832, il est le secrétaire d'une association qui vient de se créer : la Société pour l'introduction des voitures à vapeur en Belgique. Les actionnaires s'associent avec le mécanicien Jean-Chrétien Dietz, que Jobard connaît depuis plusieurs années ${ }^{23}$. Celui-ci tente de mettre au point un remorqueur de vingt-deux chevaux, mais les résultats sont mitigés. Jobard se rend en Angleterre pour y étudier le chemin de fer. Il rencontre Charles Babbage et expérimente sa machine à calculer :

" "Posez, m'a-t-il dit, à ma machine une équation de quelque degré que ce soit, elle est de force à vous répondre à la minute sans se tromper". Je le fis, et la machine, ramenée à zéro, se mit en mouvement. Chaque nombre quarré [sic] était annoncé par une cloche, une autre indiquait les racines incommensurables, et enfin l'opération terminée présentait un résultat plus parfait que ne l'eût obtenu le meilleur algébriste en une journée de travail ${ }^{24}$.»
Jobard rentre en Belgique avec plusieurs idées d'invention. Dès son retour d'Angleterre, il milite ardemment pour l'introduction du chemin de fer en Belgique et publie dans ce but la revue Le Railway. Il a compris que le train est préférable pour le développement industriel tant que les routes belges ne sont pas macadamisées : la traction sur rail présente moins de difficultés ${ }^{25}$.

Pendant ce temps, le manque de locaux place du Musée est la cause de nombreuses plaintes : le Musée des sciences naturelles s'étend (il déménagera à la suite de la découverte des iguanodons de Bernissart en 1878), le Musée de peinture et de sculpture est à l'étroit, les étudiants en droit se plaignent des odeurs provoquées par les expériences de chimie et les étudiants en littérature se plaignent de devoir croiser les cadavres que l'on apporte pour les dissections. En 1834, le squelette d'une baleine échouée à Ostende en 1827 (et que la ville de Bruxelles envisage d'acquérir) est exposé dans la plus grande salle du nouveau Palais de l'Industrie, à la grande fureur de la commission du Musée de l'Industrie qui comptait y mettre de grandes machines. Des courriers virulents sont échangés entre le bourgmestre et le ministre de $1^{\prime}$ Intérieur ${ }^{26}$. Dans Bruxelles et ses environs (édition de 1834), Augustin-Xavier Mauvy vante l'institution, toujours en pénitence dans la maison Mosselman et la recommande aux visiteurs :

" Cette collection renferme, autant que possible, tous les instruments dont il est fait mention dans les ouvrages de 'S Gravesande, Desaugulliers, Musschenbrok 
[Musschenbroek], Nollet, Sigaut de Lofond [Lafond], etc.; comme aussi dans ceux des physiciens modernes, Biot, Wollaston, Arago, Fresnel, Ampère, etc. Les instrumens [sic] ou machines sont classés d'après les différentes divisions de la physique : par exemple, d'après les propriétés générales des corps, la statique, la mécanique, l'hydrostatique, l'hydraulique, la pneumatique et les fluides impondérables. On trouve dans chacune de ces divisions ce qu'il y a de plus important dans l'histoire des inventions. Tous les instruments et appareils sont exécutés avec le plus grand soin et se trouvent dans un état qui doit satisfaire les connaisseurs.

On y voit aussi les instrumens [sic] et machines qui se rapportent à quelques parties de la mécanique, une collection de modèles de machines à vapeurs [sic]. À chaque machine se trouvent joints des modèles en coupe qui servent à montrer la structure intérieure et le jeu de piston des soupapes. Des modèles de ponts et d'écluses dont la majeure partie ont reçu leur exécution, une collection de leviers, de poulies, de cabestans, de grues, etc., dont on se sert pour l'architecture hydraulique et la construction de moulins, etc. »

Le guide du musée est Pierre Driessens qui deviendra l'un des premiers daguerréotypistes belges dès septembre 1839 . Il restera employé au Musée jusqu'à sa mort en $1849^{27}$.

Un nouvel inventaire est dressé en 1837. On y retrouve une partie des objets de 1822, notamment le mégascope : " Armoire $n^{\circ} 5,304$. Un mégascope avec deux pièces de rechange de Charles ${ }^{28}$. " Cette année-là, Jobard, qui est devenu propriétaire du quotidien Le Courrier belge, y dénonce le sort misérable réservé à l'institution. Le 25 mars 1837, il sonne l'alarme en titrant dans son journal : "Commencement de dislocation du Musée de l'État ». On distrait des objets du musée - de soi-disant doubles - pour les transporter à l'école vétérinaire et c'est un simple charpentier qui fait le tri! Jobard est devenu une personne de référence pour tout ce qui concerne les nouveautés techniques. Il innove dans son journal ${ }^{29}$ en illustrant des articles rédactionnels, appelés Bulletin industriel. Se faisant passer pour un physicien, il affirme avoir fabriqué une lampe à incandescence. Quelques jours plus tard, il avouera avoir seulement rêvé cette fabrication, car il ne disposait pas des machines nécessaires. Mais il affirme qu'il aurait pu aboutir s'il avait disposé des machines du Musée de l'Industrie!

Depuis le début des années 1830, Jobard est membre de nombreuses sociétés savantes européennes et côtoie les plus illustres savants de son temps. Son activité didactique impressionne le gouvernement qui le nomme commissaire belge à l'exposition des produits de l'industrie nationale de Paris en 1839. Il y retrouve une vieille connaissance, Alexandre von Humboldt, rencontre Louis Jacques Mandé Daguerre et discute avec l'astronome François Arago sur des changements à apporter à la législation sur les brevets d'invention en Europe.

Il envoie des articles pour ses journaux, qui seront la base de son Rapport sur l'exposition de l'industrie, publié en 
deux volumes, en 1841 et 1842 . C'est un condensé des innovations, mais aussi l'expérience de toute une vie. Jobard a développé les matières auxquelles il promet un bel avenir : machines à vapeur, filature, papier, métallurgie, sondage, industrie des bronzes, sucres, instruments de musique, éclairage, lithographie, typographie, reliure, arquebuserie et chauffage. Il est à la pointe $\mathrm{du}$ progrès, notamment avec le texte «Photographie lithographique »: il est le premier au monde à avoir pressenti que l'avenir de l'édition résidait dans les procédés photomécaniques à plat.

Il rentre début septembre en Belgique avec une chambre daguerrienne qu'il a commandée en juillet. Le 16 septembre 1839, il devient le premier photographe belge, avec une vue prise depuis sa maison, place des Barricades. Quelques jours plus tard, il devient le premier portraitiste de notre pays. Les deux clichés, présentés au Salon de 1839, ont hélas! été perdus après l'exposition ${ }^{30}$.

Jobard a développé une capacité à percevoir quelles innovations auront de l'avenir : la vapeur, l'électricité et la photographie seront les grandes affaires du XIX ${ }^{e}$ siècle! Il est un magnifique vulgarisateur, un catalyseur des changements. La Belgique adopte rapidement la photographie qui devient un des facteurs de l'accélération qui secoue cette époque. Peu après ses propres essais, il annonce, le 8 novembre 1839, que son ami, le baron Armand Séguier, est sur le point d'apporter une amélioration considérable à l'appareil photographique : « il espère pouvoir construire un nouvel appareil repliable sur lui-même, comme un accordéon ».

\section{Jobard directeur du Musée}

Les collections du Musée royal de $l^{\prime}$ Industrie ont enfin été transférées, en 1839, dans l'ancien palais de Charles de Lorraine et le musée ouvre le 27 septembre.

Un véritable travail peut être envisagé. Le 21 janvier 1841, la Chambre annonce que le gouvernement propose de porter de 25000 à 50000 francs l'allocation pour le musée, qui serait l'objet d'une réorganisation complète. L'augmentation a été défendue par le ministre de l'Intérieur Charles Liedts, à la condition toutefois que l'on procède à une réorganisation sérieuse. Il semble que le nom de Jobard circule déjà. Sa mission à l'exposition de 1839 était-elle un test de ses capa- cités? Son activité débordante à Paris et les nombreux articles enthousiastes lui ont valu une importante notoriété tant en Belgique qu'à l'étranger.

Jobard dévoile son "plan d'organisation du Musée de l'Industrie » qu'il soumet au ministre de l'Intérieur. Ce plan, publié en 1840, est accepté par les Chambres qui chargent l'auteur de son exécution. Son important réseau de relations, notamment via les nombreuses sociétés savantes étrangères dont il est membre, laisse augurer une notoriété internationale à l'institution. Le soutien du monde politique se concrétise l'année suivante. Quand Jobard est nommé directeur du Musée de l'Industrie par 
arrêté royal du 7 avril 1841, il a près de 50 ans, mais il se lance dans un nouveau combat. Il développe des conceptions muséologiques qui répondent déjà aux exigences actuelles : la conservation des objets, leur étude et l'éducation du public.

Quelques jours seulement après sa nomination, il édicte un " règlement relatif à l'exécution de levé des machines ». Il tente d'imposer pour le dessin des plans ce qu'on appellerait aujourd'hui en langage informatique une " feuille de style ». Une de ses grandes convictions est que le dessin technique codifié permettra la compréhension immédiate entre les ingénieurs de différents pays, avec pour conséquence une formidable émulation. Le musée incitera les fabricants à déposer des échantillons et des modèles de machines, et ils répondront à l'appel : en 1846, Abel Warocqué, de Mariemont, offre le modèle, qu'il fait monter par ses propres ingénieurs, de sa machine à descendre les ouvriers dans les mines.

En 1852, à la suite de l'exposition internationale de Londres de 1851, une bibliothèque est créée dans le but de présenter les nouveautés techniques et artistiques. Elle fera l'objet de publicité par voie d'affiche. Mais l'éducation du public ne se limite pas aux visiteurs : le Bulletin $d u$ Musée de l'Industrie, qui paraît de 1841 à 1883 , touche un large public belge et étranger. Des dessinateurs attachés au musée mettent leurs talents au service $\mathrm{d}$ 'inventeurs qui veulent prendre des brevets ou de fabricants qui souhaitent obtenir copie d'un plan de machine. Jobard veut que son musée soit aussi accueillant pour l'ouvrier en sarrau que pour le riche industriel.
De nombreux savants et mécaniciens gravitent autour du musée : des fabricants belges d'instruments réalisent des objets pour le musée, tels Ed. Sacré ou A. Beaulieu, lequel, sur une carte porcelaine publicitaire, se revendique "ingénieur mécanicien de l'Observatoire royal, du Dépôt de la guerre et du Musée de 1'Industrie ${ }^{31}$ ». À partir de 1843, Jobard est secondé par Polydore Lippens (18101889) ; cet ingénieux mécanicien fabrique, en 1841, « un rail-way circulaire avec un wagon en cuivre à six roues, mu par une batterie galvanique ${ }^{32} »$. En juillet 1845, Jobard importe un exemplaire $\mathrm{du}$ télégraphe Morse qui fonctionne en démonstration dans le musée. Un jeune collaborateur, le chimiste Paulin Louyet (1818-1850), meurt prématurément, victime de ses expériences sur le fluor ${ }^{33}$, bien qu'il ait lui-même conçu un masque de protection : "Masque en cuivre et verre, garni en caoutchouc, avec trompes à soupapes. Il a été construit sur un modèle de $\mathrm{M}$. Louyet, par $\mathrm{M}$. Charrière de Paris; il est destiné à protéger les organes de la respiration dans les expériences chimiques ${ }^{34}$.»

Jobard s'absorbe dans les questions de propriété intellectuelle et publie de nombreuses brochures. Il met au point une théorie économique et sociale qu'il appelle le "Monautopole ", plaidant le droit perpétuel pour les inventeurs et dénonçant les monopoles injustes. Il exige davantage d'honnêteté dans le commerce. Les félicitations viennent de toutes parts, du monde industriel et politique (le futur Napoléon III) et aussi d'intellectuels comme Victor Hugo ou Félicité de Lamennais. De 1849 à 1855, Jobard publie des articles avec l'abbé Moigno, un des plus grands vulgari- 
sateurs scientifiques de son époque. Ingénieux et fantasque, il prend luimême soixante-quinze brevets dans des domaines aussi variés que la locomotion, l'armement, le chauffage, l'acoustique, l'alimentation ou l'éclairage. En 1855, il présentera à l'exposition universelle de Paris une lampe économique qui ne brûle que pour un centime par heure, ce qui lui vaut l'appellation de « lampe du pauvre $^{35} »$. Ce visionnaire a expérimenté la transmission à distance de la musique et de la voix, prônait les maisons de fer et de verre préfabriquées et rêvait de chauffer les villes en récupérant dans les égouts la fumée des cheminées.

Jobard, ne recule devant rien pour intéresser le grand public aux progrès techniques : en 1858, il obtient un brevet d'invention " pour un théâtre industriel » qui s'inspire visiblement de spectacles comme ceux de lanterne magique, avec musiciens et bonimenteur :

" L'invention consiste à représenter les industries diverses en mouvement en exécutant effectivement les transformations de la matière première, depuis son état primitif jusqu'à celui de produits manufacturés. Ce théâtre, muni $\mathrm{d}^{\prime}$ une machine à vapeur, mettra les diverses machines en œuvre. Le prix d'entrée étant à la portée de tout le monde, permettra aux parents de conduire leurs enfants à toutes les représentations qui se feront au son de l'orchestre. Un professeur ou cicerone technologiste expliquera tous les quarts d'heure les diverses manipulations de l'industrie représentée. On donnera ainsi à la jeunesse des idées justes sur les inventions nouvelles qui seront exposées et fonctionneront effectivement sous les yeux des visiteurs. Ce théâtre sera, comme les autres, pourvu de différents rangs de loges, et n'aura rien de commun avec ces assemblages de machines muettes des expositions d'industrie, ni les expériences en petit des cabinets de physique $^{36}$. »

Jobard reste très actif jusqu'à la fin de sa vie, mais il se passionne tellement pour le spiritisme qu'il semble y laisser sa raison : Alexandre von Humboldt, dans un courrier du 2 avril 1856, regrette de le " croire engagé dans cette route ténébreuse ${ }^{37} »$. Convaincu que les grands hommes ne sont que des esprits de savants réincarnés, Jobard ira jusqu'à prétendre que la plupart de ses meilleures inventions et de ses écrits lui ont été dictés par ces esprits ${ }^{38}$. Il faut néanmoins noter que les grandes préoccupations de sa vie se retrouvent dans ses écrits sur le spiritisme : l'électricité et la photographie. Une apoplexie le terrasse, le 27 octobre 1861. En 1888, sa tombe est transférée dans le nouveau cimetière de Bruxelles, à Evere. La pierre, en forme de soufflet, est flanquée de torches renversées, symbole de la vie qui s'éteint; une étoile est symbole d'espérance et des palmes évoquent l'artiste et le savant. Sa devise y est gravée : "À chacun la propriété et la responsabilité de ses œuvres ». 


\section{Mais où est donc passé le Musée de l'Industrie?}

Hélas! l'année même de la mort de Jobard, en 1861, le Musée de l'Industrie est remis en question par les politiciens. En 1865, le ministre de l'Intérieur Vandenpeerenboom annonce qu'il espère pouvoir agrandir la salle de lecture de la Bibliothèque royale, après le transfert à Gembloux des instruments aratoires du Musée de l'Industrie ${ }^{39}$. Le 6 novembre 1866, le député Thonissen demande la suppression du musée « qui ne présente aucune utilité ». Vandenpeerenboom répond qu'il ne sera pas maintenu dans son état actuel, mais annexé à une école industrielle. On prendra par ailleurs des mesures pour faire dresser un recueil des brevets d'inventions. Le 14 octobre 1869, un arrêté royal précise la nouvelle structure :

«D'après l'article $1^{\text {er }}$, le musée royal de l'industrie comprend $1^{\circ}$ une école industrielle, $2^{\circ}$ un laboratoire de physique et de chimie, $3^{\circ}$ une bibliothèque technologique et une bibliothèque des arts industriels, $4^{\circ}$ une collection géologique ne renfermant que des substances minérales qui peuvent être utilisées dans l'industrie, $5^{\circ}$ une salle d'exposition pour les produits nouveaux et pour l'essai des appareils perfectionnés, $6^{\circ}$ une revue technologique portant le titre de Bulletin du Musée de l'industrie de Belgique. Des conférences peuvent être données au musée ${ }^{40}$.»

En janvier 1871, les travaux d'appropriation des nouveaux locaux de la Bibliothèque royale sont finalisés, au détriment du musée ${ }^{41}$. L'institution survit néanmoins et demeure un foyer d'enseignement, notamment de la photographie : des cours publics et gratuits sont données à partir de 1870 par le chimiste Léonce Rommelaere. La bibliothèque conserve des exemples des différents procédés et le Bulletin publie, de 1873 à 1883, une "Revue photographique » rédigée par Gustave De Vylder, premier président de l'Association belge de Photographie. Mais les collections ne suscitent plus d'intérêt. On en vend à l'encan une partie et le reste est transféré dans l'école industrielle établie au " Palais du Midi », qui sera ravagée par un incendie, en 1887; les derniers objets disparaissent pendant la Seconde Guerre mondiale.

J'ai cependant retrouvé des instruments agricoles dans les facultés agronomiques de Gembloux et il reste quelques objets à l'Observatoire royal de Belgique, à Uccle, car, heureusement, en 1841, l'astronome Adolphe Quetelet avait soustrait du Musée de l'Industrie une dizaine des plus beaux instruments d'astronomie; certains sont aujourd'hui les fleurons de la section des instruments scientifiques des Musées royaux d'art et $\mathrm{d}^{\prime}$ histoire de Bruxelles où ils sont en dépôt (figure 16, cahier couleur). Reste aussi un catalogue dressé par Édouard Mailly en 1846, qui recense trois mille pièces. Il permet $d$ 'imaginer ce que fut l'un des plus beaux musées techniques d'Europe au XIX ${ }^{e}$ siècle. Et peut-être de le reconstituer virtuellement? 


\section{Notes}

1. Ce personnage me passionne depuis vingt ans par son inventivité, sa curiosité et la modernité de sa vision des êtres et des choses. Je lui ai consacré ma thèse de $3^{\text {e }}$ cycle en histoire de l'art, J. B. A. M. Jobard (1792-1861), visionnaire de nouveaux rapports entre l'art et l'industrie, acteur privilégié des mutations de l'image en Belgique au XIX siècle, Louvainla-Neuve, université catholique de Louvain, 2006. Pour les 150 ans de sa mort, le 27 octobre 1861, j'ai mis en ligne le site www.jobard.eu. Mais il reste encore une vaste entreprise : la mise en ligne des catalogues et inventaires du Musée de l'Industrie; ils seront complétés par des illustrations des rares objets qui existent toujours ou, à défaut, d'objets équivalents.

2. La Haye, Algemeen Rijksarchief, Ministerie van Justitie, Lijst van personen aan welke brieven van naturalisatie zijn verleend, Inventaire 2.09.01, $\mathrm{n}^{\circ} 4876 \mathrm{~b}$. Citoyen des Pays-Bas habitant Bruxelles, il deviendra automatiquement citoyen belge après la révolution belge de 1830 .

3. Auguste Drapiez (1778-1856), naturaliste. Un fonds Drapiez est conservé à l'université MonsHainaut (bibliothèque constituée par le naturaliste contenant plus de 4000 volumes de sciences - minéralogie, chimie, géologie, botanique, zoologie), mais aussi de littérature, d'histoire, récits de voyages -, P. VAn Beneden, « Pierre-AugusteJoseph Drapiez ", Biographie nationale, Bruxelles, Académie royale de Belgique, t. 6, 1878, col. 158164.

4. Jean-Baptiste-Georges-Marie Bory de SaintVincent (1778-1846), naturaliste, géographe et dessinateur, Bulletin du bibliophile belge, t. 4, 1847, p. 419-420.

5. Jean-Baptiste Van Mons (1765-1842), chimiste, physicien, pharmacien, médecin et horticulteur, V. JACQUES, «Van Mons (Jean-Baptiste) », Biographie nationale, Bruxelles, Académie royale de Belgique, t. 15, 1899, col. 120-131.

6. Victor RAsQUIN, « Les instruments scientifiques dans les collections de Charles de Lorraine ", Archives et bibliothèques de Belgique, $\mathrm{n}^{\circ}$ spécial 66, Bruxelles, Bibliothèque royale de Belgique, 2002, p. 79-80.

7. Édouard MAILly, «Étude pour servir à l'histoire de la culture intellectuelle à Bruxelles pendant la réunion de la Belgique à la France ", Mémoires couronnés et autres mémoires, Bruxelles, Académie royale de Belgique, t. 40, 1887, p. 22-41.

8. Vol. 1, pl. 3, en regard de la p. 35.

9. Archives de la Ville de Bruxelles, Inventaire 26 (Instruction publique), dossier 103.
10. Saint-Simon était un ami très proche de sa mère qui élevait seule ses trois enfants après la mort de son mari; Charles-Étienne fut un temps son secrétaire. Il deviendra professeur à l'Université libre de Bruxelles, John BARTIER, « Des amis inconnus de Saint-Simon, les Guillery », Économies et sociétés, t. 4, 1970, p. 1199-1220.

11. C'est une ruelle étroite, entre la rue de la Montagne et la rue de l'Impératrice, qui a disparu en 1949. La Courte rue des Longs Chariots allait de la Longue rue des Longs Chariots à la rue de la Montagne (la Longue rue des Longs Chariots allant de la rue de la Putterie, près de la rue des Singes, à la rue de Loxum).

12. Michèle VAN KALCK, "Le musée et la vie culturelle à Bruxelles », Les musées royaux des beauxarts de Belgique. Deux siècles d'histoire, Bruxelles, Racine, t. 1, 2003, p. 164, note 57.

13. Le Manneken, prospectus, s.d. [mi-août 1827].

14. L'institution est en effet calquée sur le Conservatoire des arts et métiers de Paris, fondé le 19 vendémiaire an III [10 octobre 1794] par la Convention, consécutivement au discours de l'abbé Grégoire : «Je viens vous présenter les moyens de perfectionner l'industrie nationale ", le 8 vendémiaire an III [29 septembre 1794], « Le Musée des Arts et Métiers ", Beaux-Arts, hors série, 1999, p. 6, en ligne sur [http://cnum.cnam. fr/PDF/cnum_BIBL589.pdf].

15. Le Manneken, 11 octobre 1827.

16. L'Industriel ou Revue des revues, 1829, p. 247.

17. Archives de la Ville de Bruxelles, Inventaire 26 (Instruction publique), dossier 108, dossiers relatifs à la contestation entre le Gouvernement et le sieur OnderdewijngaertCanzius, ancien directeur du Musée des Arts et de l'Industrie ; musée d'armures et d'antiquités.

18. Édouard Morren, "Christian (GérardJoseph) », Biographie nationale, Bruxelles, Académie royale de Belgique, t. 4, 1873, col. 97-99.

19. Anne Desprechins, «Lievin Bauwens et sa famille ", Tablettes des Flandres, recueil 5, Bruges, 1954, p. 84.

20. Archives de la Ville de Bruxelles, fonds 26, instruction publique, boîte 108, dossier 98 ; seules des copies sont conservées, les lettres originales ayant été restituées à Napoléon Bauwens.

21. Notamment Le Belge [quotidien bruxellois] des 13, 19, 21 et 27 janvier, 8 mars et 27 juin 1832 .

22. Bibliothèque de l'Arsenal, Paris, fonds Enfantin, 7604, f $\mathrm{f}^{\circ}$ 83, lettre de Jobard à SaintAmand Bazard, 24 février 1832.

23. Jean-Chrétien Dietz, né à Zwingenberg (land van Darmstadt), est naturalisé le 4 avril 1816 (La Haye, Algemeen Rijksarchief, Ministerie van Justitie, toegang 2,09,01 inv. 4880). Le 6 septembre 
1827, Jobard publie dans Le Manneken un article avec une planche lithographique sur une nouvelle pompe inventée par Dietz (voir Catalogue Jobard). Un autre article, aussi long, paraît le 20 octobre 1827. Dietz construit également des machines pour l'extraction dans les carrières et des pompes à incendie. Son fils aîné, Charles Dietz (1801-1888) est sans doute alors déjà actif en France : il établira, en 1834, un service de véhicules à moteur entre Paris et Saint-Germain, Jacques NoIRAy, Le romancier et la machine : l'image de la machine dans le roman français, 1850-1900, Paris, Corti, 1982 t. 2, $1^{\text {re }}$ partie, "Jules Verne », p. 82; Josette Desrues, En coche, en tram, en bus... Le Paris-Saint-Germain, SaintGermain-en-Laye, Les Presses franciliennes, 2005, p. 50-52. Il est notamment l'auteur d'un modèle réduit de calèche à moteur présenté au Musée des arts et métiers-Cnam, à Paris (inv. 20482).

24. Marcellin JobARD, L'Angleterre en 1833 et 1834, Paris, [s. n.], 1834.

25 . Son action lui vaudra une lettre de remerciement des ingénieurs Gustave De Ridder et Pierre Simons, le 25 mai 1834 : ils y déclarent lui devoir en grande partie d'avoir fait triompher le principe de l'exécution des chemins de fer. Jobard présentera, en 1835, un « modèle de chemin de fer à une seule ornière ». Il prendra également un brevet pour un logophore, tube acoustique permettant la communication dans les trains. Le 13 janvier 1845, son ami Armand Séguier déposera pour lui un pli cacheté à l'Académie des sciences de Paris au sujet d'un chemin de fer électro-pneumatique.

26. Marie-Christine ClaEs, « Ne cherchez plus la baleine d'Ostende à Bruxelles : elle est à SaintPétersbourg ", Revue du Cercle d'histoire de Bruxelles et Extensions, mars 2014, p. 3-14.

27. Steven F. JosepH, Tristan SCHWILdEN et Marie-Christine CLAEs, Directory of photographers in Belgium, 1839-1905, Rotterdam, Ed. De Vries/ Antwerpen, Museum voor Fotografie, 1997, vol. 1, p. 152 ; recensement bruxellois de 1846.

28. Archives de la Ville de Bruxelles, Inventaire 26 (Instruction publique), dossier 103.

29. En 1841, il proposera une innovation encore plus étonnante : des signes typographiques émo- tionnels supplémentaires que l'on peut considérer comme les premiers "émoticônes ».

30. Au sujet des premiers daguerréotypes belges, Steven F. JosepH et Tristan ScHwILdEN, «Un cadeau à l'Europe : naissance de la photographie en Belgique », Bulletin trimestriel du Crédit communal de Belgique, $\mathrm{n}^{\circ} 168,1989$, p. 2-22.

31. Un premier répertoire des fabricants d'instruments scientifiques a été publié : Victor A. RASQUIN, Dictionnaire des constructeurs belges d'instruments scientifiques (des origines à 1914), Bruxelles, Comité national de logique, $\mathrm{d}$ 'histoire et de philosophie des sciences, 1996. J'espère mettre prochainement en ligne un répertoire des personnes liées à Jobard et au Musée de l'Industrie.

32. André CRESENS, "Polydore Lippens, acteur méconnu sur la scène du Musée de l'industrie ", actes du colloque Zénobe Gramme, Bulletin scientifique de l'association des ingénieurs électriciens sortis de l'institut Montefiore, $\mathrm{n}^{\circ}$ spécial 3-4, 2002, p. $52-78$.

33. Article nécrologique dans le quotidien bruxellois L'Indépendance, 6 mai 1850; voir aussi [http://www.chemistryexplained.com/ elements/C-K/Fluorine.html] (consulté le 15 janvier 2012).

34. Édouard MaIlly, Catalogue des collections $d u$ Musée de l'industrie, Bruxelles, Ad. Wahlen et $\mathrm{C}^{\mathrm{ie}}$, 1846, p. 238.

35. Musée des arts et métiers-Cnam, Paris, inv. 5404 .

36. Recueil spécial des brevets d'invention publié en exécution de l'art. 20 de la loi du 24 mai 1854, 4 année [1857-1858], Bruxelles, Tarlier, 1858, p. 341.

37. Lettre reproduite par L'Éclaireur, 9 avril 1856.

38. M. J.-A LuthereAu, Jobard, directeur $d u$ Musée royal de l'Industrie belge, Paris, Bureau de l'Institut polytechnique [universel], 1861, p. 51.

39. Louis Hymans, Histoire parlementaire de la Belgique de 1831 à 1880, t. 4, 1880, p. 344.

40. Bulletin du Musée de l'Industrie, août 1880, p. 1-2.

41. L'Ami de l'Ordre, 5 janvier 1871. 